\section{Rehabilitación cardiovascular y ejercicio en prevención secundaria}

\author{
MÓNICA ACEVEDO ${ }^{1}$, VERÓNICA KRÄMER ${ }^{1, a, b}$, \\ MARÍA JOSÉ BUSTAMANTE ${ }^{1, \mathrm{~b}}$, FERNANDO YÁÑEZ ${ }^{1}$, \\ DOMINIQUE GUIDI ${ }^{2, \mathrm{c}}$, RAMÓN CORBALÁN ${ }^{1}$, IVÁN GODOY ${ }^{1}$, \\ ISMAEL VERGARA ${ }^{1}$, JORGE JALIL ${ }^{1}$, MARCELO FERNÁNDEZ ${ }^{1}$
}

\section{Exercise and cardiac rehabilitation in secondary cardiovascular prevention}

Exercise and cardiac rehabilitation are indications with type I A evidence in most secondary cardiovascular prevention guidelines. Rehabilitation programs not only include exercise but also provide integral care and education about cardiovascular risk factors. However there is a paucity of such programs in Chile. Moreover there is a lack of awareness about the benefits of exercise and there is lack of knowledge about the details of exercise prescription in secondary prevention. Therefore, the divulgation of this knowledge is of utmost importance.

(Rev Med Chile 2013; 141: 1307-1314).

Key words: Cardiac care facilities; Exercise; Rehabilitation; Secondary prevention.

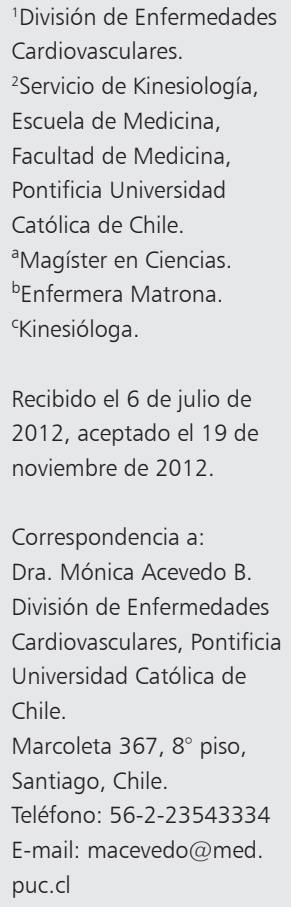

L a enfermedad cardiovascular (ECV) es la primera causa de muerte en el mundo. En las últimas décadas, si bien el tratamiento y control de algunos factores de riesgo (FR), como la hipertensión y la dislipidemia, han mejorado, el manejo de otros FR, como el sobrepeso, obesidad y sedentarismo, ha sido menos exitoso.

Estudios epidemiológicos han demostrado que la capacidad aeróbica se asocia en forma inversa y significativa a mortalidad cardiovascular y general, tanto en sujetos sanos ${ }^{1,2}$, como en prevención secundaria ${ }^{3}$. Esta relación es consistente y graduada ${ }^{2,4}$ e independiente de confusión. Basándose en la evidencia disponible, acerca de los beneficios del ejercicio en la reducción del riesgo de desarrollo y progresión de ECV, se ha recomendado la práctica de actividad física regular como una estrategia para reducir su incidencia.

A pesar de los esfuerzos para promover la actividad física, el sedentarismo sigue siendo altamente prevalente en Chile ${ }^{5}$, como en América Latina, Norteamérica y Europa ${ }^{6}$.

El objetivo de esta revisión es presentar una actualización sobre rehabilitación cardiovascular (fase II) y ejercicio en prevención cardiovascular secundaria.

\section{Rehabilitación cardiovascular y ejercicio en prevención secundaria}

Debido al avance diagnóstico y terapéutico en ECV, actualmente un mayor número de personas sobrevive a un evento cardiovascular, conllevando a una alta carga de enfermedades crónicas posteriores. Por ello, se requiere un abordaje que apoye al paciente, interviniendo los FR de manera integral, además de controles médicos y terapia farmacológica adecuada. Los programas de rehabilitación cardiovascular (RCV) incluyen estas características, influyendo además en la restauración de la calidad de vida y la mejoría de la capacidad funcional.

El rol del ejercicio en sujetos con ECV ha sido estudiado rigurosamente, con sólidos resultados en materia de seguridad, aumento de la capacidad funcional y reducción de la mortalidad cardiovascular y total ${ }^{7}$. La RCV ha sido definida por la Asociación Europea de Prevención y Rehabili- 
tación ${ }^{8}$ como la "aplicación clínica del cuidado preventivo, a través de un enfoque profesional multidisciplinario, para la reducción del riesgo integral y el cuidado global a largo plazo de los pacientes cardiovasculares". Por consiguiente, la RCV está recomendada en la actualidad con evidencia de clase I por la Sociedad Europea de Cardiología (ESC) ${ }^{9}$, la Asociación Americana del Corazón (AHA) ${ }^{9}$ y el Colegio Americano de Cardiología $(\mathrm{ACC})^{9}$ en el tratamiento de la ECV e insuficiencia cardiaca (IC).

Sin embargo, la RCV se encuentra subutilizada tanto en prevención primaria, como secundaria. De estos últimos pacientes, solamente 30\% ingresa a RCV en Europa ${ }^{8}, 15-25 \%$ en Estados Unidos de Norteamérica ${ }^{10}$ y menos de $10 \%$ en América Latina $^{11}$. Las posibles razones para esto incluyen el escepticismo médico sobre los beneficios de la RCV, la accesibilidad variable, la preferencia por indicar ejercicio en casa y el enfoque prioritario en los procedimientos de revascularización y medicación, por sobre las estrategias de cambios en los estilos de vida ${ }^{12}$. Algunos factores, dependientes del paciente son la falta de voluntad para comprometerse con el elevado número de sesiones de RCV y temas económicos, entre otros ${ }^{13-16}$.

Como una alternativa a la RCV tradicional, en entornos hospitalarios o ambulatorios, se han desarrollado otros modelos para aumentar la participación. Estos incluyen la práctica de ejercicio en la casa (con una enfermera que facilita, supervisa y monitorea el progreso) o en grupos comunitarios, dirigidos por enfermeras o kinesiólogos ${ }^{17}, y$ también existen alternativas a través de Internet, para sujetos de bajo riesgo cardiovascular.

Si bien la mayoría de los candidatos a RCV son pacientes que han tenido un infarto agudo al miocardio (IAM) o cirugía de revascularización miocárdica (CRM), la recomendación también incluye a pacientes sometidos a revascularización percutánea, trasplante de corazón o corazón/ pulmón, sujetos con angina estable, IC crónica, enfermedad vascular periférica con claudicación y pacientes sometidos a otras intervenciones quirúrgicas, como cirugías valvulares.

\section{Componentes clave de la rehabilitación cardiovascular}

Los componentes centrales de la RCV han sido resumidos en las guías de la AHA, la Asociación Americana de Rehabilitación Cardiovascular y Pulmonar $^{18}$ y la sección de Rehabilitación Cardiaca de la Asociación Europea de Prevención y Rehabilitación Cardiovascular ${ }^{8}$.

La RCV está dividida en fases, que se relacionan con el momento evolutivo de la cardiopatía. Una de las clasificaciones más usadas es la que la divide en fase I (precoz post evento u hospitalaria), fase II (activa de rehabilitación ambulatoria) y fase III (mantenimiento o alejada $)^{18}$.

Entre los elementos claves se encuentran: a) valoración del paciente; b) orientación sobre actividad física; c) entrenamiento físico; d) orientación sobre nutrición; e) control de diabetes; f) control de peso; g) control de dislipidemia; h) control de presión arterial (PA), i) orientación sobre cese tabáquico y j) manejo psicosocial. En esta revisión nos centraremos en el entrenamiento físico.

La actividad física se define como cualquier movimiento corporal producido por la contracción músculo-esquelética, que ocasiona un gasto energético superior al basal.

El entrenamiento físico corresponde a la ejecución de movimientos corporales planificada, estructurada y repetitivamente para mantener o mejorar uno o más atributos de la condición física ${ }^{8}$. Por lo tanto, se refiere a una intervención estructurada con una duración determinada.

Antes de comenzar la práctica de entrenamiento físico, se debe evaluar el riesgo de complicaciones cardiovasculares e identificar sujetos potencialmente inestables ${ }^{19}$. Para esto, y para determinar el nivel de supervisión requerida, la mayoría de los candidatos a RCV debiera ser sometida a un TE limitado por síntomas. Este debiera ser realizado posterior a 14 días del evento coronario agudo. En los sujetos en los que no se pueda realizar un TE, debiera considerarse una evaluación sub máxima o un test de marcha de 6 min. Todos los programas estructurados de RCV debieran incluir una supervisión profesional que abarque: examen físico, evaluación de síntomas asociados al ejercicio o arritmias, y monitorización de la frecuencia cardiaca y la PA antes, durante y después del ejercicio. Los resultados esperados de la RCV son: aumento de la capacidad aeróbica, realce de la flexibilidad, mejoramiento de la resistencia y fuerza muscular, respuestas fisiológicas atenuadas a pruebas físicas y mejoramiento de la calidad de vida. 


\section{Evidencia de ensayos clínicos}

La mayoría de la información disponible acerca de ejercicio en prevención secundaria proviene de pacientes con enfermedad coronaria. Con respecto a la RCV en esos sujetos, Cochrane reportó en 2001 , en más de 8.000 pacientes, $27 \%$ de reducción de mortalidad general en el grupo con ejercicio como intervención exclusiva (OR 0,73; CI: 0,54$0,98)$. La mortalidad cardiaca disminuyó en $31 \%$ (OR 0,69; CI: $0,51-0,94$ ) y en $26 \%$ (OR 0,74; CI: 0,57-0,96) en los grupos de ejercicio solo y RCV integral como intervención, respectivamente ${ }^{20}$. No hubo efectos en IAM recurrente no fatal. Este metaanálisis, sin embargo, incluyó predominantemente hombres de edad mediana y bajo riesgo cardiovascular.

Una revisión más reciente, que incluyó 48 estudios, con aproximadamente 9.000 pacientes, mostró una reducción de $20 \%$ en la mortalidad general y $16 \%$ en mortalidad CV, en sujetos en $\mathrm{RCV}$ versus tratamiento habitual. En este estudio, tampoco hubo diferencias significativas en las tasas de IAM no fatal y revascularización ${ }^{19}$.

En el año 2005, Clark y cols. ${ }^{21}$ reportaron en un metaanálisis de 63 ensayos en más de 20.000 pacientes con enfermedad coronaria, una reducción de mortalidad general de $15 \%$ (13\% a 12 meses y $47 \%$ a 24$),$ y $17 \%$ de reducción en la recurrencia de IAM a 12 meses.

La RCV también beneficia a otros pacientes de prevención secundaria. Un ensayo clínico controlado de RCV versus tratamiento estándar en sujetos posterior a CRM, demostró significativamente menos eventos $\mathrm{CV}$ en los sujetos en $\mathrm{RCV}$ (18\% versus 35\%), además de una reducción en las re-hospitalizaciones a 10 años ${ }^{22}$.

Por otra parte, es sabido que el ejercicio programado aumenta la tolerancia al esfuerzo físico en los pacientes con angina estable, incrementando el umbral de angina y retrasando el comienzo de la isquemia. Hambrecht y cols. ${ }^{23}$ compararon, en un estudio de 12 meses aleatorio y controlado, en 101 pacientes hombres, los efectos del entrenamiento físico versus la angioplastia con stent: el ejercicio se asoció a una tasa de sobrevida libre de eventos de $88 \%$ versus $70 \%$ en revascularizados con stent, además de mejor capacidad aeróbica y reducción de costos.

Con respecto a los sujetos con IC estable, un metaanálisis sobre entrenamiento físico en 2.387 pacientes reportó mejoría en la capacidad funcional, reducción de la sintomatología y tendencia al aumento en la sobrevida (OR 0,98; CI: 0,61-1,32). No se reportaron muertes relacionadas con el ejercicio en más de $60.000 \mathrm{~h} /$ paciente de entrenamiento físico ${ }^{24}$. Finalmente, el reciente estudio HF- ACTION (Heart Failure: A Controlled Trial Investigating Outcomes of Exercise TraiNing), en 2.331 sujetos con IC estable, distribuidos en forma aleatoria a tratamiento estándar más entrenamiento aeróbico programado versus tratamiento estándar, no demostró mejoría significativa en el punto final combinado, compuesto por mortalidad y hospitalización (HR 0,93; CI: 0,84-1,02). Uno de los principales problemas en este estudio fue la falta de adherencia al ejercicio, la que disminuyó en forma significativa en el seguimiento.

\section{Efectos cardioprotectores del entrenamiento físico (Figura 1)}

El entrenamiento físico tiene varios efectos cardioprotectores: aumento de la tolerancia al ejercicio, disminución de los síntomas cardiacos, efectos positivos sobre los lípidos, mejoría de factores psicosociales como ansiedad, estrés y cese tabáquico, y reducción de la mortalidad general y $\mathrm{CV}^{7}$.

La RCV tiene efectos favorables sobre los FR cardiovascular ${ }^{10,25}$. El efecto más conocido es el cambio en los lípidos, aumentando 8-23\% el colesterol HDL y 5-26\% la relación colesterol total a HDL y reduciendo los triglicéridos hasta en $22 \%$. El entrenamiento físico también mejora la sensibilidad a la insulina y disminuye modestamente el peso corporal y la masa grasa, reduciendo el riesgo de desarrollar diabetes tipo $2^{26}$. Además influencia positivamente la $\mathrm{PA}$, reduciéndola $4-9 \mathrm{~mm} / \mathrm{Hg}$ en promedio.

El entrenamiento físico también ha demostrado tener efectos hemodinámicos positivos en los sujetos con enfermedad coronaria, disminuyendo la frecuencia cardiaca, la PA y el consumo de oxígeno máximo (en promedio 20\%). Estos efectos son mayores en los sujetos más mal acondicionados ${ }^{27}$. El entrenamiento, además, puede aumentar el flujo coronario por aumento de la distensibilidad o elasticidad y por la vasodilatación coronaria dependiente del endotelio ${ }^{28}$. Como ejemplo, Hambrecht y cols., demostraron un aumento sig- 


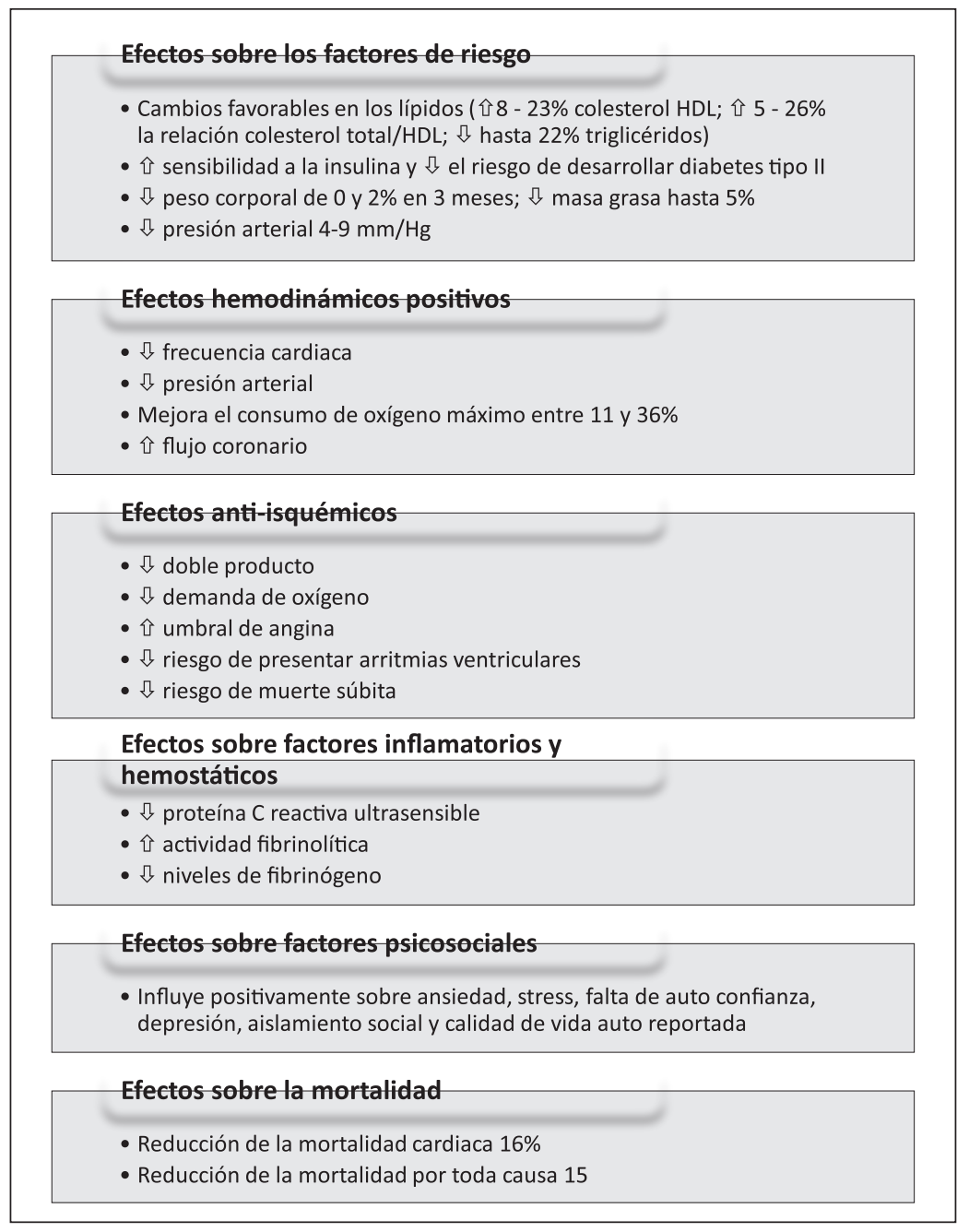

Figura 1. Efectos cardioprotectores del entrenamiento físico. $\hat{\imath}=$ aumento; $\sqrt{\Omega}=$ disminución. nificativo en la dilatación arterial dependiente del endotelio en pacientes con enfermedad coronaria y disfunción endotelial, luego de cuatro semanas de entrenamiento físico vigoroso de resistencia ${ }^{29}$.

Por otro lado, el ejercicio de resistencia también tiene efectos anti-isquémicos ${ }^{30}$ : en sujetos con enfermedad coronaria avanzada, el ejercicio puede producir preacondicionamiento isquémico, incrementando la tolerancia miocárdica a la isquemia y reduciendo así el riesgo de presentar arritmias malignas ${ }^{27,31}$. El ejercicio también reduce la actividad simpática y aumenta la parasimpática, pudiendo reducir el riesgo de muerte súbita ${ }^{28}$.

Otro efecto beneficioso del entrenamiento físico es la mejoría de factores inflamatorios y hemostáticos: disminuye la proteína C-reactiva, aumenta la actividad fibrinolítica del plasma y puede reducir los niveles de fibrinógeno ${ }^{25,32}$.

Finalmente, desde la perspectiva del paciente, los efectos más destacados de la RCV se relacionan a factores psicosociales: mejora la ansiedad, estrés, falta de autoconfianza, depresión, aislamiento social y calidad de vida ${ }^{10}$.

\section{Seguridad del entrenamiento físico}

Existen múltiples guías para la prescripción de ejercicio aeróbico y de resistencia en pacientes con $\mathrm{ECV}^{27,33,34}$.

Se ha demostrado que la incidencia de eventos cardiovasculares mayores y muerte súbita durante 
ejercicio vigoroso es baja: 1 en 50.000-100.000 h/ paciente eventos cardiovasculares mayores (incluyendo IAM y PCR reanimado), y 2 en 1,5 millones de $\mathrm{h} /$ paciente fallece ${ }^{7,35}$. Sin embargo, es importante recalcar que el ejercicio vigoroso aumenta el riesgo de IAM. Al respecto, la estratificación de riesgo previa al ejercicio ha ayudado a identificar a los pacientes más vulnerables de sufrir eventos asociados al ejercicio, requiriendo estos una monitorización más intensiva y mayor supervisión médica durante sus sesiones ${ }^{36}$. Esta estratificación se detalla a continuación:

- Clase A: sujetos aparentemente sanos, que tienen bajo riesgo cardiovascular asociado al ejercicio.

- Clase B: sujetos con enfermedad coronaria estable, por lo tanto, con bajo riesgo de sufrir complicaciones al practicar ejercicio vigoroso.

- Clase C: sujetos con riesgo cardiovascular elevado (los que han tenido IAM múltiples o PCR, los que pertenecen a la clasificación NYHA clase III o IV, los que presentan una capacidad aeróbica de menos de 6 equivalentes metabólicos o isquemia inducida por TE.

- Clase D: pacientes con contraindicación absoluta de realizar ejercicio por inestabilidad hemodinámica. Al respecto, los sujetos de Clase C debieran participar en programas de RCV con supervisión médica por al menos 8-12 semanas, antes de remitirlos a un programa en la comunidad o su hogar.

\section{Aspectos claves para prescribir un programa de ejercicios en prevención secundaria:}

\section{Entrenamiento aeróbico (Figura 2)}

El riesgo de complicaciones cardiovasculares y ortopédicas aumenta a mayor riesgo individual de ECV, como a mayor intensidad del ejercicio. Por ello, todo sujeto debiera ser sometido a una evaluación rigurosa antes de incorporarse a RCV. Esto es indispensable en sujetos en prevención secundaria.

Todo sujeto debiera someterse a un TE limitado por síntomas. Este proporciona información mucho más allá de la simple alteración del ECG. El TE nos muestra presencia o ausencia de desviaciones significativas del segmento ST, cambios en el ECG o angina de esfuerzo, el nivel máximo de METS alcanzado, el doble producto al comienzo de los cambios del ST o de la sintomatología, la frecuencia cardiaca y PA durante el ejercicio y comienzo o exacerbación de las arritmias, entre otros. En pacientes en prevención secundaria es importante considerar la respuesta al ejercicio en relación a la terapia medicamentosa. Al respecto, es deseable que los pacientes que realizan un TE para prescripción de ejercicio no suspendan su terapia con beta bloqueadores y nitratos previo a la prueba, para así evaluar la respuesta cronotrópica y hemodinámica ${ }^{37}$. Finalmente, la mayoría de los nomogramas utilizados para estimar la capacidad aeróbica máxima, se basan en individuos jóvenes y sanos, que se han ejercitado sin sujetarse de la barra de la cinta caminadora. Por lo tanto, en todos los sujetos que se afirmen de la barra frontal durante el TE, se debiera restar por lo menos 1 MET a la capacidad aeróbica máxima para prescribir

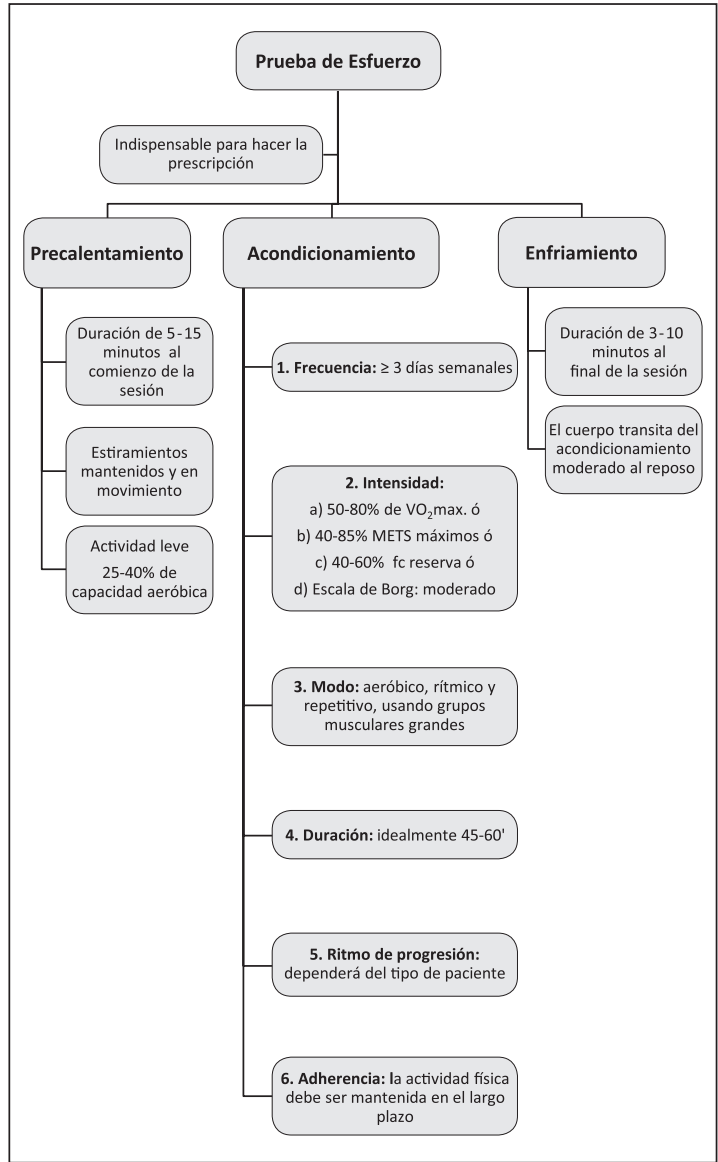

Figura 2. Prescripción de entrenamiento aeróbico. $\mathrm{VO}_{2} \max =$ consumo de oxígeno máximo; $\mathrm{METS}=$ equivalentes metabólicos; fc $=$ frecuencia cardiaca. 
ejercicio, ya que la capacidad funcional estará sobre estimada.

Una vez determinada la capacidad aeróbica máxima, se deben abordar los siguientes componentes para una prescripción de $\mathrm{RCV}^{38}$ : precalentamiento, acondicionamiento y enfriamiento (Figura 2).

Precalentamiento: Debe durar 5-15 min, para evitar lesiones ortopédicas y permitir la adaptación hemodinámica y fisiológica al esfuerzo. Debiera incluir estiramientos mantenidos y en movimiento, así como actividad aeróbica leve (25-40\% de la capacidad aeróbica del sujeto).

Acondicionamiento: Corresponde a la etapa más importante de la sesión, debiera estar dirigido por seis aspectos claves:

1. Frecuencia: las guías recomiendan realizar ejercicio en la mayoría de los días de la semana para lograr los beneficios cardiovasculares, disminuir el riesgo CV y manejar el peso. Sin embargo, de manera general, se debiera entrenar por lo menos 3 días semanales. A niveles de intensidad de entrenamiento más bajos, la frecuencia debiera ser de 5-7 días semanales, para alcanzar un mayor gasto calórico.

2. Intensidad: este es el componente más difícil de prescribir. Lo recomendado es alcanzar una intensidad "moderada". El enfoque general es prescribir la intensidad basándose en alguna de las siguientes características: a) $50-80 \%$ del consumo de oxígeno máximo; b) 40-85\% de los METS máximos o c) 40-60\% de la frecuencia cardiaca de reserva. Pero la intensidad también puede fundarse en un enfoque subjetivo, de acuerdo a la percepción subjetiva de cansancio del sujeto, método también llamado Escala de Borg ${ }^{39}$. Esta escala estipula un valor numérico de acuerdo al nivel de cansancio expresado por el sujeto durante la práctica de ejercicio.

3. Modo: la recomendación es prescribir actividad física aeróbica, rítmica y repetitiva, promoviendo el uso de grupos musculares grandes al nivel de intensidad requerido.

4. Duración: por lo menos 20-30 (idealmente 45-60) min de actividad física aeróbica. Una de las metas de la mayoría de los programas de ejercicios debe ser aumentar en $2.000 \mathrm{kcal}$ el gasto calórico semanal. Sin embargo, la acumulación de sesiones cortas, de 15 min o más, ha demostrado los mismos beneficios que sesiones más largas.

5. Ritmo de progresión: dependerá de la historia médica del paciente, su nivel basal de acondicionamiento, comorbilidades y edad.

6. Adherencia: la clave del programa será que la actividad física sea mantenida en el largo plazo. La tasa de adherencia de los programas de RCV en Estados Unidos de Norteamérica es de $50-75 \%$ a los 6 meses.

Enfriamiento: Corresponde a un período de 3-10 min, durante el cual el cuerpo transita del acondicionamiento moderado al reposo. Este período no debiera concluir abruptamente, sino que gradualmente, previniendo la disminución del retorno venoso por estasis venoso, la hipotensión y las potenciales arritmias asociadas al aumento abrupto de las catecolaminas post ejercicio inmediato.

\section{Ejercicios de resistencia}

Los ejercicios de resistencia tienen como objetivo aumentar la fuerza, potencia y resistencia muscular mediante el levantamiento de pesas ${ }^{8}$.

Clásicamente, los programas de ejercicio han enfatizado la práctica de ejercicios dinámicos del tren inferior. Sin embargo, está demostrado que un ejercicio de resistencia suave a moderado puede mejorar efectivamente la fuerza y resistencia muscular, ayudando a prevenir y manejar distintas patologías médicas, además de promover el control de los FR cardiovascular y aumentar el bienestar psicosocial. Además, este tipo de ejercicio ha demostrado atenuar el doble producto, independientemente de su intensidad ${ }^{33}$.

El entrenamiento de fuerza es especialmente importante en pacientes más frágiles, en los que los ejercicios aeróbicos pueden dificultarse por debilidad muscular. Por otra parte, los pacientes con ECV deben realizar los ejercicios de fuerza con una monitorización cuidadosa de síntomas cardiovasculares adversos.

Lamentablemente, existe poca evidencia publicada sobre ejercicios de resistencia. En general, la recomendación es ejercitar 2-3 veces semanales, un set de 12-15 repeticiones a 30-40\% del máximo logrado en una repetición del tren superior, y a $40-50 \%$ del tren inferior ${ }^{8,38}$. 


\section{Conclusión}

La RCV es un componente clave en los pacientes en prevención secundaria. El ejercicio no sólo confiere beneficios $\mathrm{CV}$, sino que también mejora la calidad de vida. Aún es necesario, sin embargo, contar con más estudios randomizados en RCV para demostrar la reducción en la mortalidad en estos pacientes. Debiéramos aspirar a que en Chile se incorpore la RCV en el tratamiento de todo sujeto con ECV.

\section{Referencias}

1. Blair SN, Kohl HW 3rd, Paffenbarger RS Jr, Clark DG, Cooper KH, Gibbons LW. Physical fitness and all-cause mortality. A prospective study of healthy men and women. JAMA 1989; 262: 2395-401.

2. Manson JE, Greenland P, LaCroix AZ, Stefanick ML, Mouton CP, Oberman A, et al. Walking compared with vigorous exercise for the prevention of cardiovascular events in women. N Engl J Med 2002; 347: 716-25.

3. Myers J, Prakash M, Froelicher V, Do D, Partington S, Atwood JE. Exercise capacity and mortality among men referred for exercise testing. N Engl J Med 2002; 346: 793-801.

4. Lee IM, Hsieh CC, Paffenbarger RS, Jr. Exercise intensity and longevity in men. The Harvard alumni health study. JAMA 1995; 273: 1179-84.

5. Ministerio de Salud PUC y UAH. Encuesta nacional de salud. Chile 2009-2010. 2011.

6. Schargrodsky H, Hernández-Hernández R, Champagne BM, Silva H, Vinueza R, Silva Aycaguer LC, et al. Carmela: Assessment of cardiovascular risk in seven latin american cities. Am J Med 2008; 121: 58-65.

7. Wenger NK. Current status of cardiac rehabilitation. J Am Coll Cardiol 2008; 51: 1619-31.

8. Corra U, Piepoli MF, Carre F, Heuschmann P, Hoffmann $\mathrm{U}$, Verschuren $\mathrm{M}$, et al. Secondary prevention through cardiac rehabilitation: Physical activity counselling and exercise training: Key components of the position paper from the cardiac rehabilitation section of the european association of cardiovascular prevention and rehabilitation. Eur Heart J 2010; 31: 1967-74.

9. Van de Werf F, Bax J, Betriu A, Blomstrom-Lundqvist C, Crea F, Falk V, et al. Management of acute myocardial infarction in patients presenting with persistent stsegment elevation: The task force on the management of st-segment elevation acute myocardial infarction of the european society of cardiology. Eur Heart J 2008; 29:
2909-45.

10. Ades PA. Cardiac rehabilitation and secondary prevention of coronary heart disease. N Engl J Med 2001; 345: 892-902.

11. Korenfeld Y, Mendoza-Bastidas C, Saavedra L, MonteroGómez A, Pérez-Terzic C, Thomas RJ, et al. Current status of cardiac rehabilitation in Latin America and the Caribbean. Am Heart J 2009; 158: 480-7.

12. Suaya JA, Stason WB, Ades PA, Normand SL, Shepard DS. Cardiac rehabilitation and survival in older coronary patients. J Am Coll Cardiol 2009; 54: 25-33.

13. Evenson KR, Rosamond WD, Luepker RV. Predictors of outpatient cardiac rehabilitation utilization: The minnesota heart surgery registry. J Cardiopulm Rehabil 1998; 18: 192-8.

14. Evenson KR, Fleury J. Barriers to outpatient cardiac rehabilitation participation and adherence. J Cardiopulm Rehabil 2000; 20: 241-6.

15. Lane D, Carroll D, Ring C, Beevers DG, Lip GY. Predictors of attendance at cardiac rehabilitation after myocardial infarction. J Psychosom Res 2001; 51: 497-501.

16. Cooper AF, Jackson G, Weinman J, Horne R. Factors associated with cardiac rehabilitation attendance: A systematic review of the literature. Clin Rehabil 2002; 16: 541-52.

17. Haskell WL. Cardiovascular disease prevention and lifestyle interventions: Effectiveness and efficacy. J Cardiovasc Nurs 2003; 18: 245-55.

18. Balady GJ, Williams MA, Ades PA, Bittner V, Comoss P, Foody JM, et al. Core components of cardiac rehabilitation/secondary prevention programs: 2007 update: A scientific statement from the american heart association exercise, cardiac rehabilitation, and prevention committee, the council on clinical cardiology; the councils on cardiovascular nursing, epidemiology and prevention, and nutrition, physical activity, and metabolism; and the american association of cardiovascular and pulmonary rehabilitation. Circulation 2007; 115: 2675-82.

19. Taylor RS, Brown A, Ebrahim S, Jolliffe J, Noorani H, Rees K, et al. Exercise-based rehabilitation for patients with coronary heart disease: Systematic review and meta-analysis of randomized controlled trials. Am J Med 2004; 116: 682-92.

20. Jolliffe JA, Rees K, Taylor RS, Thompson D, Oldridge N, Ebrahim S. Exercise-based rehabilitation for coronary heart disease. Cochrane Database Syst Rev. 2001:CD001800.

21. Clark AM, Hartling L, Vandermeer B, McAlister FA. Meta-analysis: Secondary prevention programs for patients with coronary artery disease. Ann Intern Med 2005; 143: 659-72. 
Rehabilitación cardiovascular y ejercicio en prevención secundaria - M. Acevedo et al

22. Hedback B, Perk J, Hornblad M, Ohlsson U. Cardiac rehabilitation after coronary artery bypass surgery: 10year results on mortality, morbidity and readmissions to hospital. J Cardiovasc Risk 2001; 8: 153-8.

23. Hambrecht R, Walther C, Mobius-Winkler S, Gielen S, Linke A, Conradi K, et al. Percutaneous coronary angioplasty compared with exercise training in patients with stable coronary artery disease: A randomized trial. Circulation 2004; 109: 1371-8.

24. Smart N, Marwick TH. Exercise training for patients with heart failure: A systematic review of factors that improve mortality and morbidity. Am J Med 2004; 116 : 693-706.

25. Kramer V, Acevedo M, Orellana L, Chamorro G, Corbalán R, Bustamante MJ, et al. [Association between cardiorespiratory fitness and cardiovascular risk factors in healthy individuals]. Rev Med Chile 2009; 137: 737-45.

26. Knowler WC, Barrett-Connor E, Fowler SE, Hamman RF, Lachin JM, Walker EA, et al. Reduction in the incidence of type 2 diabetes with lifestyle intervention or metformin. N Engl J Med 2002; 346: 393-403.

27. Leon AS, Franklin BA, Costa F, Balady GJ, Berra KA, Stewart KJ, et al. Cardiac rehabilitation and secondary prevention of coronary heart disease: An american heart association scientific statement from the council on clinical cardiology (subcommittee on exercise, cardiac rehabilitation, and prevention) and the council on nutrition, physical activity, and metabolism (subcommittee on physical activity), in collaboration with the american association of cardiovascular and pulmonary rehabilitation. Circulation 2005; 111: 369-76.

28. Hambrecht R, Adams V, Erbs S, Linke A, Krankel N, Shu $\mathrm{Y}$, et al. Regular physical activity improves endothelial function in patients with coronary artery disease by increasing phosphorylation of endothelial nitric oxide synthase. Circulation 2003; 107: 3152-8.

29. Hambrecht R, Wolf A, Gielen S, Linke A, Hofer J, Erbs S, et al. Effect of exercise on coronary endothelial function in patients with coronary artery disease. N Engl J Med 2000; 342: 454-60.

30. Hosokawa S, Hiasa Y, Takahashi T, Itoh S. Effect of regu- lar exercise on coronary endothelial function in patients with recent myocardial infarction. Circ J 2003; 67: 221-4.

31. Bolli R. The late phase of preconditioning. Circ Res 2000; 87: 972-83.

32. Mora S, Cook N, Buring JE, Ridker PM, Lee IM. Physical activity and reduced risk of cardiovascular events: Potential mediating mechanisms. Circulation 2007; 116: 2110-8.

33. Pollock ML, Franklin BA, Balady GJ, Chaitman BL, Fleg JL, Fletcher B, et al. Aha science advisory. Resistance exercise in individuals with and without cardiovascular disease: Benefits, rationale, safety, and prescription: An advisory from the committee on exercise, rehabilitation, and prevention, council on clinical cardiology, american heart association; position paper endorsed by the american college of sports medicine. Circulation. 2000; 101: 828-33.

34. Fletcher GF, Balady GJ, Amsterdam EA, Chaitman B, Eckel R, Fleg J, et al. Exercise standards for testing and training: A statement for healthcare professionals from the american heart association. Circulation 2001; 104: 1694-740.

35. Franklin BA, Bonzheim K, Gordon S, Timmis GC. Safety of medically supervised outpatient cardiac rehabilitation exercise therapy: A 16-year follow-up. Chest 1998; 114: 902-6.

36. Wenger NK, Froelicher ES, Smith LK, Philip A, Ades PA, Berra K, et al. Clinical practice guidelines no. 17: Cardiac rehabilitation as secondary prevention. Rockville, md: Us department of health and human services, public health service, agency for health care policy and research, national heart, lung and blood institute; 1995. Ahcpr publication 96-0672.

37. Foody JM. Preventive cardiology: Strategies for the prevention and treatment of coronary artery disease. Totowa, N.J.: Humana Press; 2001.

38. Thompson PD. Exercise prescription and proscription for patients with coronary artery disease. Circulation. 2005; 112: 2354-63.

39. Borg GA. Psychophysical bases of perceived exertion. Med Sci Sports Exerc 1982; 14: 377-81. 